\title{
Monostotic Fibrous Dysplasia of the Middle Phalanx of the Hand
}

\author{
Lars Maruschke ${ }^{\mathrm{a}, \mathrm{d}}$, Tobias Baumann ${ }^{\mathrm{a}}$, Horst Zajonc ${ }^{\mathrm{b}}$, Georg Herget ${ }^{\mathrm{c}}$
}

\begin{abstract}
Monostotic fibrous dysplasia is a rare lesion of the upper extremity, especially the hand. We present the case of a 20 -year-old male patient with fibrous dysplasia of the middle phalanx of the right ring finger. The initial diagnosis was made by X-ray examination and magnetic resonance imaging (MRI). Histopathologic confirmation was obtained during definitive surgical treatment with curettage and bone grafting.
\end{abstract}

Keywords: Monostotic fibrous dysplasia; Tumor-like lesion; Middle phalanx; Fibrous dysplasia

\section{Introduction}

Fibrous dysplasia is best understood as a dysplastic anomaly of bone-forming mesenchymal tissue [1]. The hallmark of the disease is an inability of bone-forming tissue to produce mature lamellar bone and an arrest at the level of woven bone [1].

Monostotic fibrous dysplasia (MFD) has been well documented in rib, skull, jaw bones, femur and tibia. Only a few cases of MFD in the upper extremity have been reported. Among these metacarpals [2,3] and phalanges [4-7] were most commonly involved.

\footnotetext{
Manuscript accepted for publication March 21, 2013

${ }^{a}$ Department of Diagnostic Radiology, University Medical Centre Freiburg, Freiburg, Germany

${ }^{\mathrm{b}}$ Department of Plastic and Hand Surgery, University Medical Centre Freiburg, Freiburg, Germany

${ }^{c}$ Department of Orthopaedics and Traumatology, University Medical Centre Freiburg, Freiburg, Germany

${ }^{\mathrm{d}}$ Corresponding author: Lars Maruschke, University Hospital Freiburg, Department of Diagnostic Radiology, Hugstetter Str. 55, 79104

Freiburg, Germany. Email: lars.maruschke@uniklinik-freiburg.de

doi: http://dx.doi.org/10.4021/jmc1146w
}

Clinically, patients usually present with swelling and deformation of the affected limb, sometimes combined with pain und functional disorder.

This report presents an unusual occurrence of fibrous dysplasia at the middle phalanx of the right hand.

\section{Case Report}

A 20-year-old adult presented with spindle deformity of the right ring finger. He reported that swelling of the finger had been present for several years. Due to the recent onset of pain, the patient was referred to our outpatient clinic. Physical examination additionally revealed restricted mobility and painful range of motion of the distal interphalangeal joint.

The initially performed X-ray examination showed a distended middle phalanx with ground glass appearance. Cortical thinning and involvement of the adjacent joints could be observed (Fig. 1). The T1 weighted magnetic resonance image showed a homogeneously low signal of the lesion (Fig. 2 ), whereas the short-tau turbo inversion recovery image (STIR) demonstrated a signal area isointense to normal bone marrow (Fig. 3). The osseous lesion exhibited a strong signal enhancement after contrast agent injection (Fig. 4). No signs of soft tissue involvement could be noted.

As imaging findings were compatible with MFD, curettage was performed and the defect was grafted using autologous bone from the distal radius.

Histopathology revealed osseous metaplasia with an abundance of fibroblastic tissue and immature trabeculae of woven bone (Fig. 5), characteristic for fibrous dysplasia [8].

Postoperative follow-up revealed consolidation of the grafted bone and no recurrence (Fig. 6). Full range of finger motion was regained.

\section{Discussion}

Fibrous dysplasia (FD) has been described as a medullar fibro-osseous lesion that can involve a single site (monostotic) or multiple locations (polyostotic). FD can be considered a dysplastic anomaly of bone-forming mesenchymal tissue 


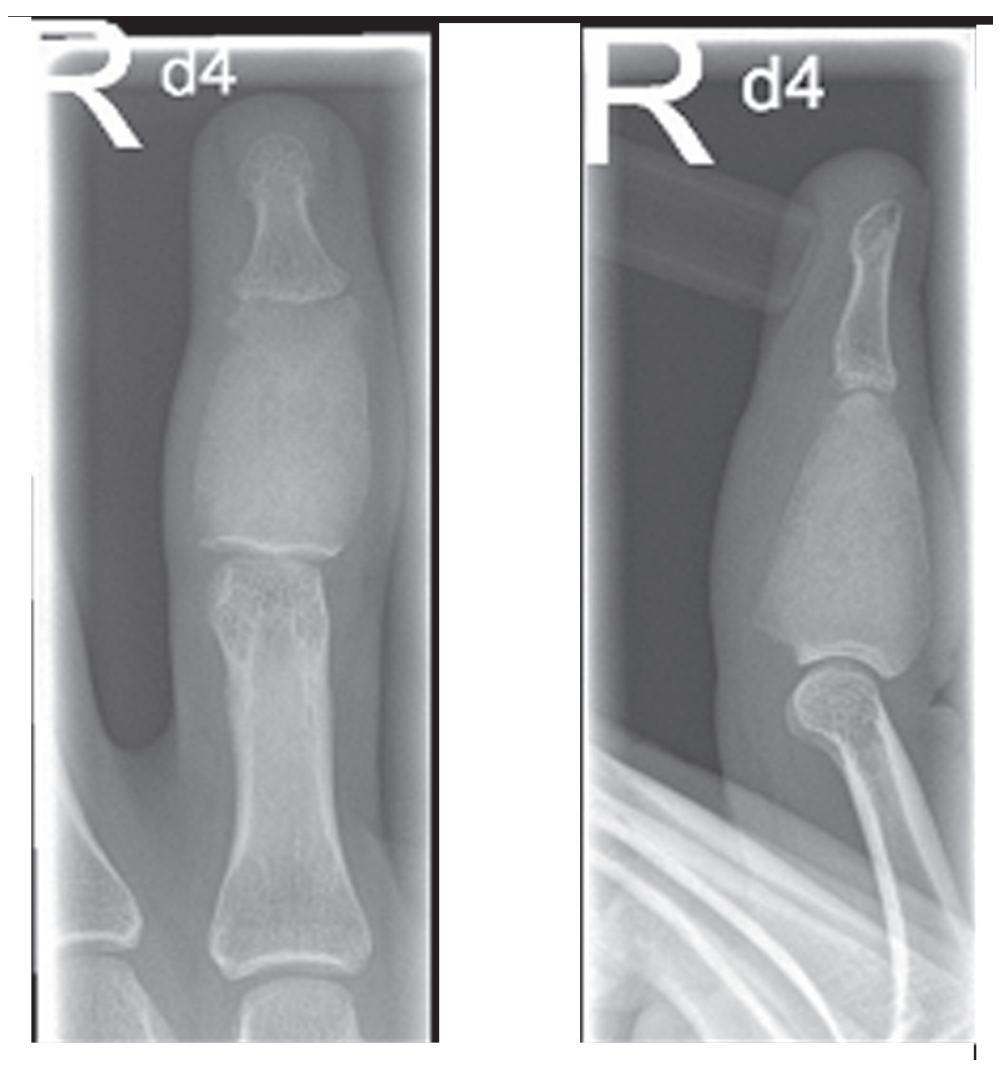

Figure 1. X-ray of the right ring finger: Distended middle phalanx with a ground glass opacity and thinned cortex.

with an inability of bone-forming tissue to produce mature lamellar bone [1].

Recent investigations suggest a genetic background of the disease that leads to an impaired cell differentiation into mature osteocytes, which would be capable of producing

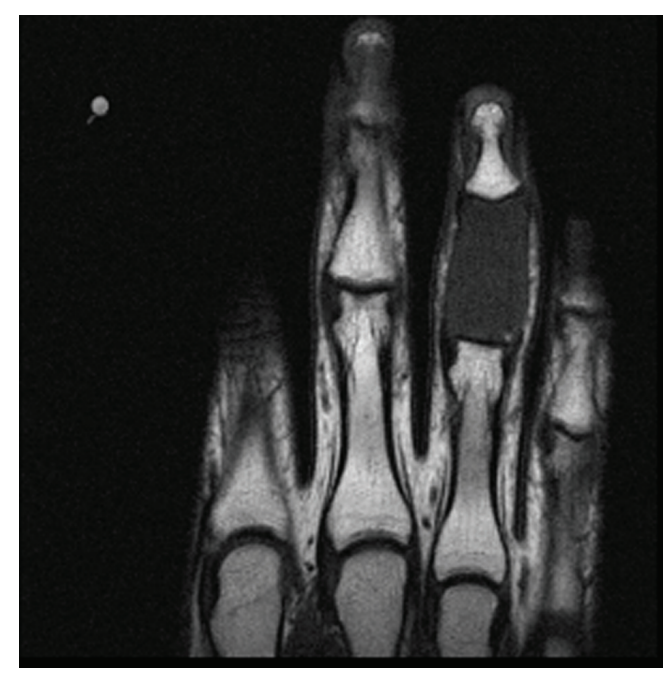

Figure 2. T1 weighted MRI: homogeneous low signal of the middle phalanx of D IV. lamellar bone. The associated mutations affect signal-transducing $\mathrm{G}$ proteins and have been implicated in the development of multiple endocrinopathies in McCune-Albright syndrome and soft tissue related myxomas in patients with Mazabraud syndrome. Fibrous dysplasia is part of both men-

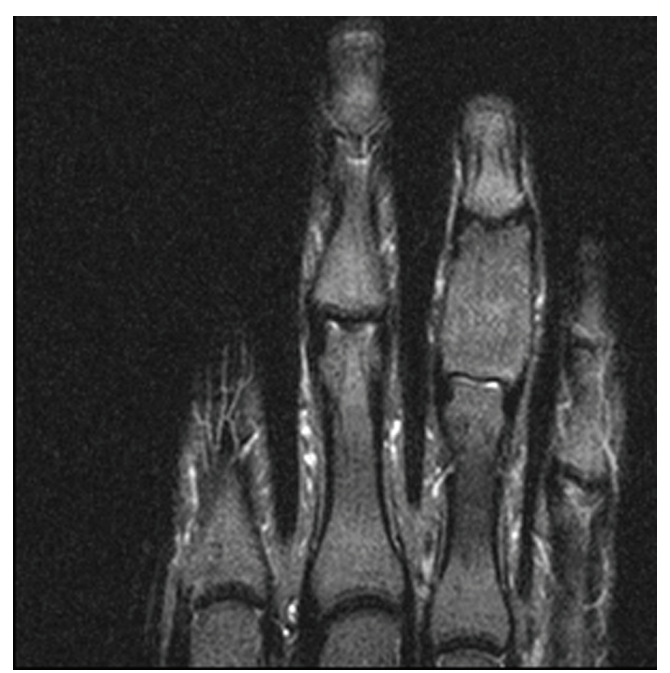

Figure 3. TIRM: isointense signal area of the middle phalanx of D IV. 


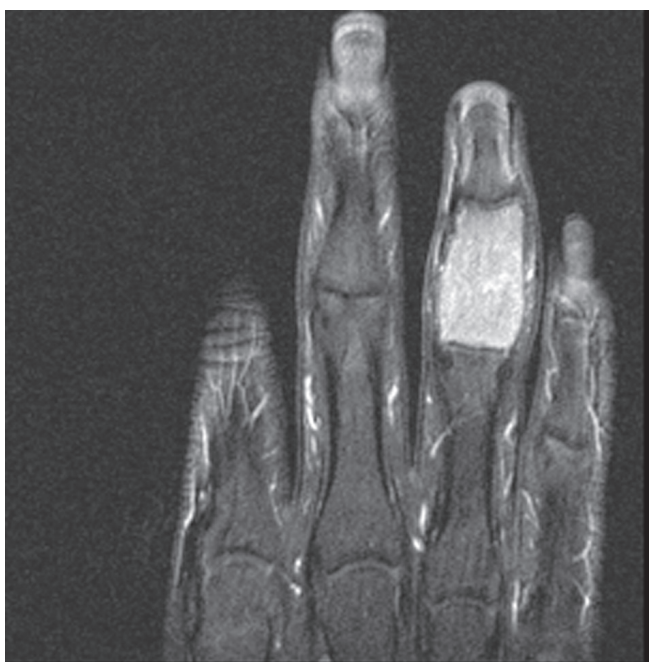

Figure 4. T1 weighted fat saturated contrast enhanced MRI with intensive signal alteration.

tioned diseases [1].

FD accounts for $7-10 \%$ of benign bone tumors and tumor-like lesion, respectively. FD is most commonly a disease of young children and adolescents that usually regresses after cessation of bone growth. The monostotic form is 6 times more frequent than the polyostotic form and tends to manifest later in the life [9]. The lesion can cause deformity, pain and sometimes fracture.

Most frequently the proximal femur, the skull and the ribs are affected [10]. Involvement of the upper extremity, especially the phalanx, is extremely rare with only a few cases having been reported in the literature. On plain film radiography phalangeal FD presents as a lobulated or expanded and sometimes distorted phalanx with ground glass appearance with intact cortex $[5,6]$. Alternatively, FD can present as a lytic cyst-like lesion with incomplete periostal reaction [11]. Lesions have a variable $\mathrm{X}$-ray attenuation depending on their degree of mineralization and bony spicules [12]. FD of

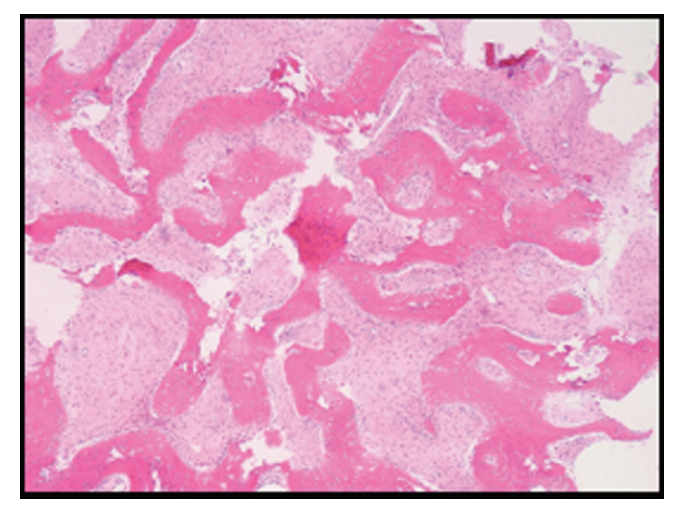

Figure 5. Osseous metaplasia with an abundance of fibroblastic tissue and immature trabeculae of woven bone. the skull is usually expansive with a ground glass appearance $(56 \%)$, may have an homogeneously dense $(23 \%)$ or cystlike pattern (21\%) [9]. Furthermore, lesions are associated with endosteal scalloping, with or without bone expansion and thinning of the cortex. Generally there is no soft tissue extension or periostal reaction unless a fracture or malignant transformation occurs [1]. Malignant transformation into chondroblastic osteosarcoma has been reported in less than $1 \%$ of cases [13].

The MRI appearance of this lesion, as documented in previous cases, shows an isointense signal area and a high signal area on the T2 weighted sequence [6], a homogeneous low signal on T1-weighted images and a homogeneous isointense signal on STIR images.

Furthermore, we found a considerable enhancement in the $\mathrm{T} 1$ weighted fat saturated sequence (T1WI fs) after injection of contrast agent. Whereas cortical disruption was suspected on plain film radiography, this finding could neither be confirmed by MRI nor during surgery.

Surgery is indicated for confirmatory biopsy, correction of deformity, prevention of pathologic fracture, and/or removal of symptomatic lesions. The use of cortical grafts is preferred over cancellous grafts or bone-graft substitutes because of the superior physical qualities of remodelled cortical bone [14].

Benign differential diagnoses of lytic lesions of the phalanx include enchondroma, solitary bone cyst (SBC), aneurysmal bone cyst $(\mathrm{ABC})$, giant cell reparative granuloma and brown-tumor-hyperparathyroidism (HPTH). Important malignant differential diagnosis comprises osteosarcoma and metastases $[15,16]$. Infectious diseases like tubercular dactylitis (spina ventosa) may also show a comparable disease pattern [17].

Enchondroma is an intramedullary lesion with welldefined borders that usually has low-intermediate signal on T1WI and intermediate on Proton density-weighted image (PDWI). On T2WI and fat saturated T2WI it has a predominantly high signal. Enchondroma shows enhancement of contrast agent, however, in varying patterns [15].

Solitary bone cysts often have a peripheral rim of low signal on T1WI a T2WI adjacent to normal medullary bone and usually contain fluid. Thin peripheral contrast enhancement can be seen at the margins of the lesion [15].

Aneurysmal bone cyst, often excentrically located, has a low signal rim on T1WI and T2WI adjacent to normal medullary bone or to extraosseous soft tissue. There are various combinations of low, intermediate, and/or high signal on T1WI, PDWI and T2WI. Fluid-fluid levels and internal separations can, however, be considered as highly indicative of ABC. Moreover, variable contrast enhancement can be seen that commonly involves the margins and the internal septae [15].

The giant cell reparative granuloma can have heterogeneous low, intermediate, and/or high signal a T1WI, PDWI 


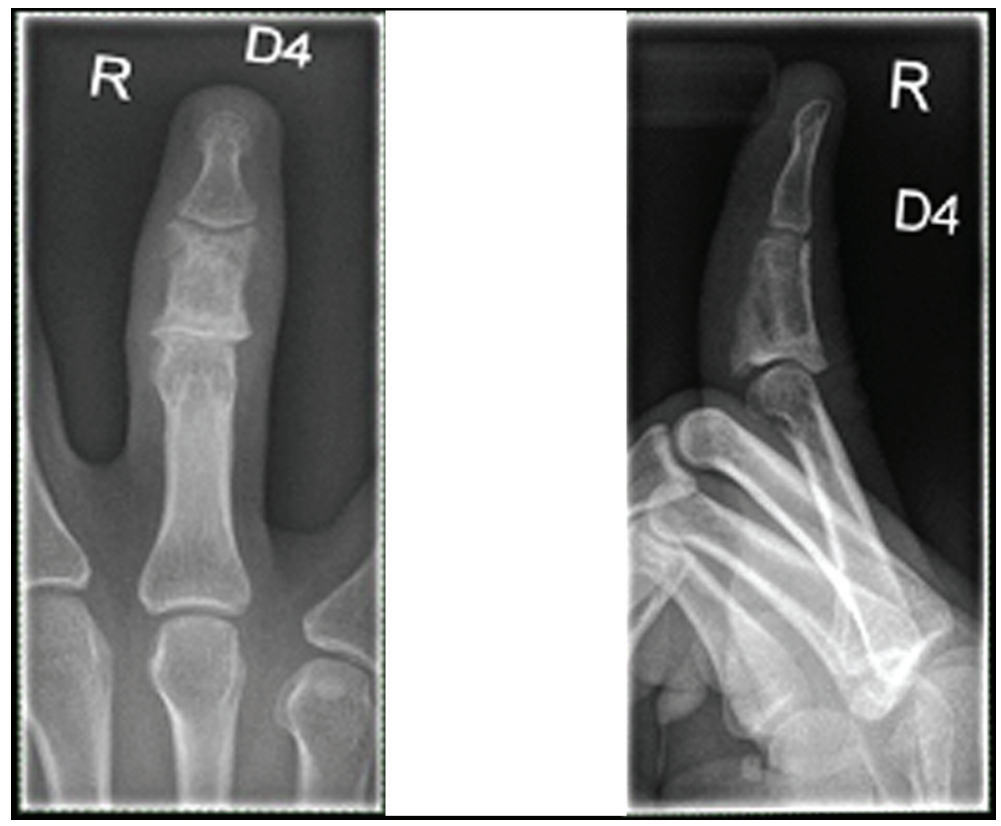

Figure 6. X-ray: 8 months after surgery.

and T2WI. Furthermore peripheral rim-like and central Gdcontrast enhancement can be seen.

The brown-tumor-hyperparathyroidism (HPTH) often has a low-intermediate signal on T1WI and an intermediate or slightly high signal on T2WI. It typically shows contrast enhancement. Zones of low signal on T2WI may occur due to hemosiderin [18].

Osteosarcoma is an intramedullary malignant lesion which shows low-intermediate signal on T1WI and mixed low, intermediate or high signal on T2WI. Usually it contains matrix mineralization and ossification with low signal on T2WI. Furthermore, it usually shows contrast enhancement. Zones of cortical destruction are typically seen, when tumor extends into the extraosseous soft tissue [15].

Metastatic lesions appear as single or multiple osteolytic lesions. Lesions are well-circumscribed, although poorly defined margins can occur with highly aggressive tumors. They are often associated with cortical destruction and extraosseous extension. Metastases usually show contrast enhancement. Periostal reaction is uncommon [15].

The tubercular dactylitis is an unusual form of skeletal tuberculosis of the short bones. The main radiographic feature is a cystic expansion. The lesion reveals alterated marrow signal, which is hypointensive in T1WI and hyperintensive in T2WI. Furthermore, the cortex often appears irregular and sometimes is surrounded by a soft tissue abscess $[17,19]$.

\section{Conclusion}

Although it is very rare, MFD should be included in differ- ential diagnosis of lytic lesions of the phalanges. Its typical $\mathrm{X}$-ray features comprise ground glass attenuation, cortical thinning and distortion. MRI is helpful in the differential diagnosis, especially to rule out soft tissue components.

\section{Competing Interests}

The authors declare that they have no competing interests.

\section{References}

1. Dorfman HD. New knowledge of fibro-osseous lesions of bone. Int J Surg Pathol. 2010;18(3 Suppl):62S-65S.

2. Di Giovanni C, Rosa MA. [On an unusual case of monostotic fibrous dysplasia of metacarpal localization]. Arch Putti Chir Organi Mov. 1983;33:451-454.

3. Ross DW, Vitale CC. Monostotic fibrous dysplasia of the metacarpal; a case report. J Bone Joint Surg Am. 1955;37-A(1):196-200.

4. Mailander P, Schaller E. [Fibrous bone dysplasia of the proximal phalanx of the middle finger. Description of the disease picture based on a case report]. Chirurg. 1986;57(2):109-110.

5. Borys D, Canter R, James MA. Monostotic fibrous dysplasia of the distal phalanx: case report. J Hand Surg Am. 2010;35(8):1294-1296.

6. Hatanaka H. Monostotic fibrous dysplasia of the middle phalanx. Hand Surg. 2007;12(2):91-95.

7. Milliez PY, Thomine JM. Rare benign bone tumors and 
dystrophy in the hand. Review of literature and report of four cases. Ann Chir Main. 1988;7(3):189-201.

8. Cohen, B.M. Fibrous dysplasia. Oral surgery, oral medicine, and oral pathology 12, 66-76 (1959).

9. Meyers, P. Fibrous Dysplasia. Vol. MRI of Bone and Soft Tissue Tumors and Tumorlike Lesions 449-455 (Thieme, 2007).

10. Pollandt K, Engels C, Werner M, Delling G. [Fibrous dysplasia]. Pathologe. 2002;23(5):351-356.

11. Amillo S, Schweitzer D, San Julian M. Monostotic fibrous dysplasia in the hand: a case report. J Hand Surg Am. 1996;21(2):290-292.

12. Dorfman HD, C.B. Bone tumors. The Mosby Publisher, 441-481 (1998).

13. Kaushik S, Smoker WR, Frable WJ. Malignant transformation of fibrous dysplasia into chondroblastic osteosarcoma. Skeletal Radiol. 2002;31(2):103-106.

14. DiCaprio MR, Enneking WF. Fibrous dysplasia. Patho- physiology, evaluation, and treatment. J Bone Joint Surg Am. 2005;87(8):1848-1864.

15. Meyers, P. Osseous tumors and tumorlike lesions at the hands and the feet. Vol. MRI of Bone and Soft Tissue Tumors and Tumorlike Lesions 199-209 (Thieme, 2007).

16. Komano, B., Pudas. Localized Bone Lesions. Vol. Differential Diagnosis in Conventional Radiology 80,125 (Thieme, 2007).

17. Patra SR. Tubercular dactylitis (spina ventosa). Postgrad Med J. 2009;85(1010):699-700.

18. Meyers, P. Intramedullary lesions associated with cortical destruction and extraosseous extension. Vol. MRI of Bone and Soft Tissue Tumors and Tumorlike Lesions 130 (Thieme, 2007).

19. Gyanshankar PM, Dhamgaye TM, Amol BF. Spina ventosa discharging tubercle bacilli--a case report. Indian $\mathrm{J}$ Tuberc. 2009;56(2):100-103. 